\title{
Phylogeny and adaptive evolution of the brain-development gene microcephalin (MCPH1) in cetaceans
}

Michael R McGowen ${ }^{1,2^{*}}$, Stephen H Montgomery ${ }^{3}$, Clay Clark ${ }^{1}$ and John Gatesy ${ }^{1}$

\begin{abstract}
Background: Representatives of Cetacea have the greatest absolute brain size among animals, and the largest relative brain size aside from humans. Despite this, genes implicated in the evolution of large brain size in primates have yet to be surveyed in cetaceans.

Results: We sequenced $~ 1240$ basepairs of the brain development gene microcephalin (MCPH1) in 38 cetacean species. Alignments of these data and a published complete sequence from Tursiops truncatus with primate MCPH1 were utilized in phylogenetic analyses and to estimate $\omega$ (rate of nonsynonymous substitution/rate of synonymous substitution) using site and branch models of molecular evolution. We also tested the hypothesis that selection on MCPH1 was correlated with brain size in cetaceans using a continuous regression analysis that accounted for phylogenetic history. Our analyses revealed widespread signals of adaptive evolution in the MCPH1 of Cetacea and in other subclades of Mammalia, however, there was not a significant positive association between $\omega$ and brain size within Cetacea.

Conclusion: In conjunction with a recent study of Primates, we find no evidence to support an association between MCPH1 evolution and the evolution of brain size in highly encephalized mammalian species. Our finding of significant positive selection in MCPH1 may be linked to other functions of the gene.
\end{abstract}

\section{Background}

The human brain is arguably one of the most remarkable adaptations in the history of life. Compared to other mammals, the human lineage has undergone a massive expansion in relative brain and forebrain size, cortical surface area, and overall cognitive ability [1]. However, many other vertebrates exhibit increased relative brain and forebrain sizes, as well as complex social and cognitive behaviours. For example, odontocete cetaceans (toothed whales) have some of the largest brains relative to their body mass among extant mammals [2]. Relative brain size in some odontocete species is greater than non-human primates [3], and in absolute terms, the giant sperm whale (Physeter macrocephalus) has the largest brain of any living organism at a maximum of 10 $\mathrm{kg}$ [4]. According to some researchers, high relative

\footnotetext{
* Correspondence: mmcgowen@med.wayne.edu

'Department of Biology, University of California, Riverside, Riverside, CA 92521 USA

Full list of author information is available at the end of the article
}

brain or forebrain sizes are positively correlated with indices of cognition or "intelligence" [1,5], although this association has been criticized in the literature [6,7].

Among extant cetacean species, absolute and relative brain size vary widely (Figure 1). There is some evidence that a large shift towards increased brain size took place near the base of Odontoceti (toothed whales), and a further increase in Delphinoidea, the group that includes Delphinidae (oceanic dolphins) among others [8]. Delphinids display the greatest encephalization and the most complex behavior among cetaceans $[9,10]$. The evolution of large brains in odontocetes has been linked to their intricate behavioral repertoire and to their use of echolocation [11], which requires production and processing of high frequency sounds to perceive spatial relationships in the surrounding liquid environment [2]. Odontocete cetaceans also are distinguished by indices of complex cognition that are convergent with many primate species $[9,12]$. Some researchers have proposed that odontocetes evolved large brains for
C Biomed Central

(ㄷ) 2011 McGowen et al; licensee BioMed Central Ltd. This is an Open Access article distributed under the terms of the Creative Commons Attribution License (http://creativecommons.org/licenses/by/2.0), which permits unrestricted use, distribution, and reproduction in any medium, provided the original work is properly cited. 


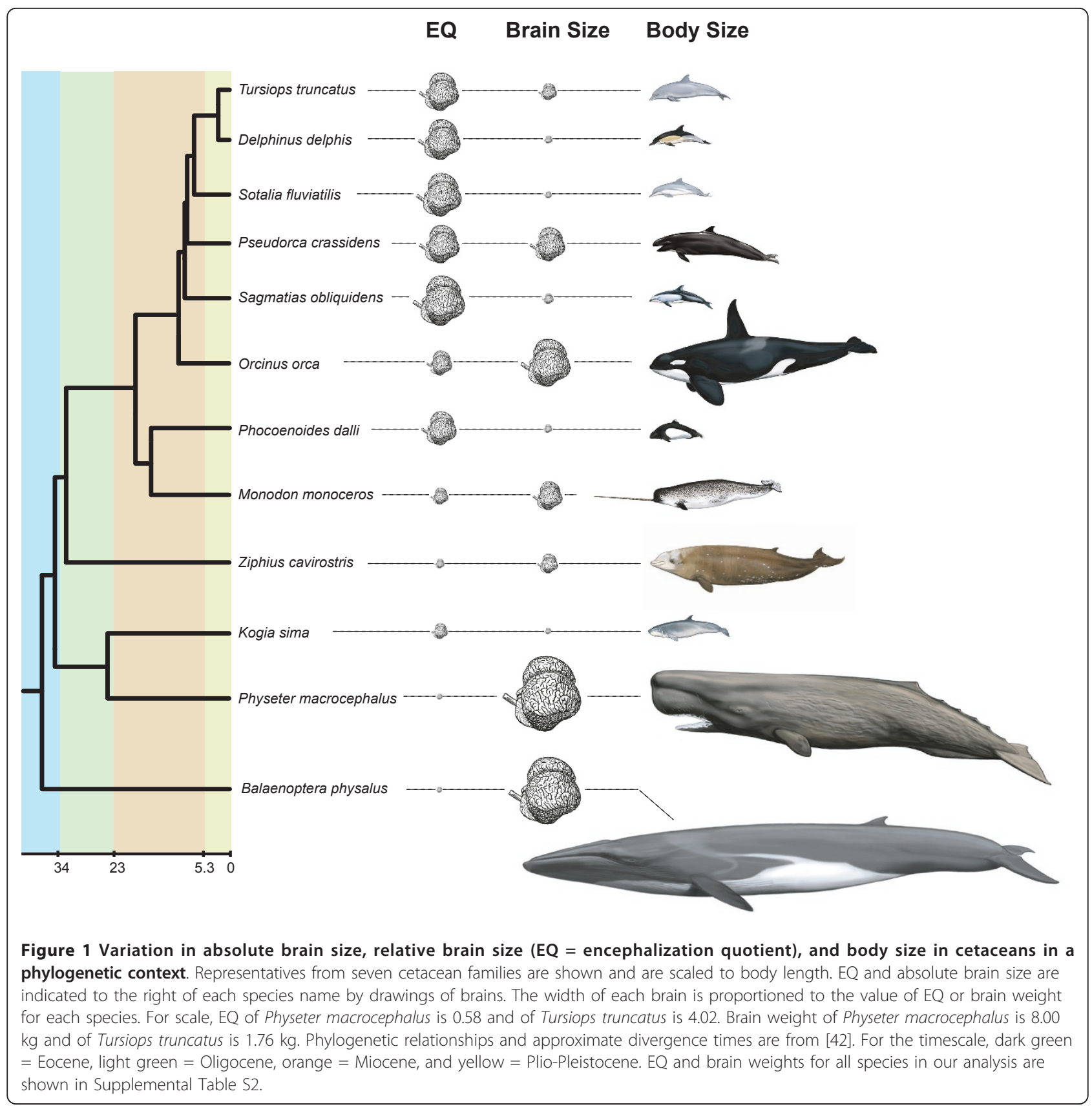

thermoregulation and are not as socially and behaviorally advanced as primates [13], however this has been contested [10].

In primates, researchers have documented the association of six genes (MCPH1, ASPM, CDK5RAP2, CENPJ, STIL, WDR62) with the human congenital disorder of primary microcephaly, a disease marked by a two-thirds reduction in brain size and moderate to severe mental retardation [14-22]. All six microcephaly genes identified thus far are involved in control of the neural cell cycle and/or centrosome function, and therefore have a potentially large impact on the proliferation of neural precursor cells [23,24]. Comparative analyses of proteincoding DNA sequences from primates suggest that these genes have evolved adaptively in primates [14-19,25].

Multiple recent studies have revealed the importance of one of these genes, microcephalin (MCPH1), in maintaining genomic stability through mediation of the response to double-strand DNA breakage and regulation of chromosome condensation in the cell cycle [26-31]. $M C P H 1$ is expressed in multiple tissues including those 
of the brain, liver, and kidney, but shows particularly high expression in neural progenitor cells of the forebrain [32]. Microcephaly genes may play a role during development in switching between symmetric and asymmetric mitosis of neural progenitor cells to produce neurons in the cerebral cortex [24,33]. Specifically, microcephalin may affect the first stage of neural cell division, with decreased $\mathrm{MCPH} 1$ function causing premature mitotic entry, eventually leading to a reduced pool of neural progenitor cells [24]. In addition, the developing neuronal tissue in the brain seems particularly vulnerable to apoptosis as a result of DNA damage caused by double-strand breaks, thus potentially bringing about a decrease in viable neurons for individuals with impaired MCPH1 function [34,35].

Although some lineages of whales and dolphins have experienced profound evolutionary increases in relative brain size, selection on microcephaly genes has not been investigated in detail within Cetacea. Several researchers have indicated the potential value of comparisons between brain genes of primates and other large-brained species for understanding processes underlying neurological novelty in primates including humans $[23,33,36]$. Here we sequenced a large segment of microcephalin (MCPH1) from 38 cetacean species, including 34 toothed whales and four baleen whales, and compared these data with published sequences from primates, which have convergently evolved large brain size. We also downloaded complete $M C P H 1$ protein-coding sequences from Ensembl, including representatives of major primate lineages as well as the common bottlenose dolphin, Tursiops truncatus. We enumerated patterns of site- and branch-specific selection in cetacean $M C P H 1$ and compared these estimates with patterns of selection intensity in primates and other mammals. We also explicitly tested the hypothesis that brain size is positively correlated with the intensity of selection at the nucleotide level in $M C P H 1$ following the approach taken by [25].

\section{Methods}

\section{Sampling and DNA Amplification}

We sampled 38 species of cetaceans from nine families; twenty-three species belonged to Delphinidae, a family that includes the highest relative brain sizes as measured by the encephalization quotient (EQ) [10]. We also included members of Mysticeti (baleen whales) and Physeteridae (giant sperm whale) that represent taxa with the largest absolute brain sizes that have ever evolved $[4,9,10]$. All cetacean taxa included in this study are listed in Additional File 1: Table S1.

We designed primers for exon 8 of $M C P H 1$ from an alignment of sequences from the 2.0 build of the Bos taurus (domestic cow) genome and genomic data for
Tursiops truncatus available via Genbank. Exon 8 consists of the highly variable inter-BRCT domain sequence (IBS) and makes up approximately half of the coding region of $M C P H 1$. The IBS region shows evidence of positive selection on the lineage leading to humans $[16,19]$. A small portion of intron 7 and most of exon 8 (total amplicon $=\sim 1237$ basepairs $[\mathrm{bp}]$ ) were PCR amplified using MCPH1INT7F1 (5' GCT TTA TCA CGT TAT GGG CGG AC 3') or MCPH1INT7F2 (5' GCT TTA TCA CGT TAT GGG CGG ACT G 3') in the forward direction and MCPH1EX8R1 (5' GAG AGA CCA GTA AAG GAG GTT CAC 3'), MCPH1EX8R2 (5' AGG AGG TTC ACA TAC TTT CAC TAC 3'), or MCPH1RSeq2 (5' CGG GAG AAA AGT AAT CAT CG $\left.3^{\prime}\right)$ in the reverse direction. PCR products were sequenced using the above primers, as well as MCPH1F1 (5' AAA ACG AGA AGT GTC CGT CCG C 3'), MCPH1F2 (5' CCT GTC TGC TAC GCC ATC TGT AAC 3'), MCPH1FSeq3 (5' TTT CCA GGA GAG AGA GGA CC 3'), MCPH1R1 (5' TTT CCA CAT CCC AGT CGC CTA C 3'), and MCPH1RSeq1 (5' TCT CCT TGA GAT TAT CGG G 3'). PCR was performed using $1 \mu \mathrm{l}$ template DNA, 100 pmol of each primer, $1 \mathrm{X}$ AccuPrime PCR Buffer I (Invitrogen), and 1 unit AccuPrime Taq DNA Polymerase High Fidelity (Invitrogen) in a 50 $\mu \mathrm{l}$ reaction. PCR conditions consisted of 45 cycles of 1 min denaturation at $94^{\circ} \mathrm{C}, 1 \mathrm{~min}$ annealing at $58^{\circ} \mathrm{C}$, and 1 min elongation at $68^{\circ} \mathrm{C}$. All new sequences were deposited in Genbank (accession numbers HQ873570HQ873608).

\section{Data Set Compilation and Alignment}

Three data sets were assembled. To investigate selection pressure over the whole gene, the "whole-gene" data set was compiled using complete $M C P H 1$ coding sequences downloaded via Ensembl (Homo, Pan, Pongo, Macaca, Callithrix, Tupaia, Rattus, Mus, Canis, Equus, Bos, and the delphinid cetacean, Tursiops). Species were selected due to phylogenetic position, completeness, and quality of available sequence. We also compiled a data set consisting of sequences derived from exon 8 and a small segment of intron 7 (the "exon 8" data set), including those sequenced here and sequences downloaded for a wider range of species, largely primates, from the Ensembl and Genbank databases. Genbank accession numbers for all downloaded sequences are listed in Additional File 1. The third data set ("reduced exon 8") consisted of a reduced set of taxa that excluded sequences that were less than $90 \%$ complete (e.g., Balaenoptera acutorostrata, Kogia sima) and sequences from species belonging to Monodontidae due to the presence of a stop codon near the end of the sequence (see Additional File 1). Sequences for all data sets were aligned using CLUSTAL W [37] with a gap-opening penalty of 
10 and a gap-extension penalty of 1 . Exonic indels were multiples of $3 \mathrm{bp}$ and were adjusted by eye to reflect the open reading frame.

\section{Phylogenetic Analyses}

Please see Additional File 1 for a full account of phylogenetic methods.

\section{Tests for Positive Selection and Selective Constraints}

Positive selection acting on the complete coding sequence of $M C P H 1$ was examined by estimating $\omega$ $(d N / d S$, the ratio of the rate of nonsynonymous substitution to the rate of synonymous substitution) using the site models in the codeml program of PAML 4.0 [38]. An unrooted species tree of Boreoeutheria [39-41] was used as an input tree with Rodentia, Primates + Scandentia, and Laurasiatheria positioned as a basal polytomy. Model M1a (nearly-neutral: $\omega_{0}<1, \omega_{1}=1$ ) was compared to M2a (positive selection: $\omega_{0}<1, \omega_{1}=1, \omega_{2}$ $>1$ ) and M8a (nearly neutral; beta distribution: $0<\omega_{0}<$ 1 and $\omega_{1}=1$ ) was compared to model M8 (positive selection: beta distribution: $0<\omega_{0}<1$ and $\omega_{1}>1$ ) by performing likelihood ratio tests (LRTs) and assessing their significance using a $\chi^{2}$ distribution (two degrees of freedom [df] for M1a vs. M2a; one df for M8 vs. M8a). For the comparison of M8 vs. M8a, we halved the pvalue as suggested by [38]. A Bayes Empirical Bayes (BEB) analysis was implemented to calculate posterior probabilities of positively selected sites using the M2a and M8 models as described in [38]. An individual site was considered to have undergone positive selection ( $\omega$ $>1$ ), if the posterior probability was $\geq 0.95$. Variation in $\omega$ among branches was examined using the free-ratio model in which each branch of the tree was given a separate $\omega$-value. The fit of the free-ratio model was compared to model M0 in which all branches in the tree were assigned the same $\omega$ value using the LRT with 20 degrees of freedom for the whole $M C P H 1$ data set.

To further investigate selection on exon 8 of $M C P H 1$, for which 38 cetacean species were sequenced (Additional File 1: Table S1), all intronic sequence was deleted, as well as sequence downstream of a stop codon found near the 3' end of exon 8 in monodontids (see Additional File 1). All "exon 8" analyses were implemented using a species tree and also a gene tree derived from phylogenetic analysis of the $M C P H 1$ data. The species tree employed was a composite gathered from several sources [39-42]; the gene tree for PAML analyses was the optimal topology recovered by maximum likelihood (ML) analysis and was consistent with the 50\% majority rule consensus of Bayesian trees. In both the species tree and the gene tree, Loxodonta, Euarchontoglires, and Laurasiatheria were treated as a basal trichotomy. The ratio $\omega$ was then estimated in the codeml program of PAML 4.0 [38], as described above for the complete coding sequence of $M C P H 1$. Separate analyses were conducted using the site models for all mammals, cetaceans only, odontocetes only, delphinids only, mysticetes only, primates only, and all mammals excepting cetaceans and primates. By analyzing various subclades of mammals using the site models, the goal was to determine whether evidence for positive selection is a general feature of $M C P H 1$ in mammals, or is instead restricted to only certain mammalian lineages. In addition, analyses using the species tree and gene tree were also conducted for the "reduced exon 8" data set (see above).

For the taxon-rich exon 8 alignment, $\omega$ was also estimated for individual branches and groups of branches [38]. Several branch model analyses were conducted including a free-ratio model (all branches separate), two-ratio models in which one branch was given a separate $\omega$ (repeated for the branch leading to the last common ancestor [LCA] to each of the following groups: Cetacea, Mysticeti, Odontoceti, Delphinoidea, Delphinidae, as well as each of the terminal branches leading to Physeter and Orcinus), and two-ratio models in which one stem-based clade was given a separate $\omega$ (repeated for Cetacea, Mysticeti, Odontoceti, Delphinoidea, and Delphinidae). All models listed above were tested against a one-ratio model (M0) using LRTs and $1 \mathrm{df}$. Branch models with $p \leq 0.004(0.05 / 13)$ after Bonferroni correction for multiple tests were interpreted as having a significantly different $\omega$ on the "foreground" branches of interest in comparison to the "background" $\omega$ on all remaining branches of the tree. Comparisons between background branches and foreground branch(es) at the base of lineages or within whole clades were conducted to test whether the pattern of selection on these branches was significantly distinct from the rest of the tree. Branches were selected for comparison due to their proposed relation to an evolutionary change in relative and/or absolute brain size. For example, in the case of Odontoceti and Delphinoidea, Marino et al. [8] proposed that these clades mark shifts associated with increases in relative brain size. Delphinidae was selected due to the high relative brain size of multiple species within the clade [8]. The branches leading to Mysticeti, Physeter, and Orcinus were also tested because these lineages terminate at species with large absolute brain sizes [8,9] (Figure 1, Additional File 1: Table S2). For all PAML branch models, both a species tree and the $M C P H 1$ gene tree were used as input trees.

\section{Variation of $\mathrm{MCPH} 1$ within Cetacean Species}

We recorded heterozygous sites for each cetacean $M C P H 1$ sequence that was generated in this study. Sites in sequencing chromatograms that showed nearly equal 
height peaks for two different bases at the same position were considered true heterozygous sites (i.e., due to divergent alleles that were PCR-amplified from that specimen). The position (first, second, or third codon) and the nature (synonymous or nonsynonymous, transition or transversion, etc.) of change at each heterozygous site were noted, and this variation within species was compared to the pattern of nucleotide substitution in MCPH1 between cetacean species. For Tursiops truncatus and Delphinus capensis, we compared sequences derived from two individuals of each species and checked for intraspecific variation in MCPH1.

\section{Analysis of Associations Between $\omega$ and Phenotype}

To test prior hypotheses of association between $\omega$ and phenotype, we compiled data on absolute brain and body mass for 27 cetaceans for which we had molecular data; these traits vary widely among extant cetaceans (Figure 1). Absolute brain and body mass data came from multiple, previously published sources $[8,9,43,44]$. For Platanista minor, we used measurements derived from the very close relative, Platanista gangetica [42,43]. Because some body masses above came from individual animals and may not represent the full size range of a particular species, we also gathered data on maximum body size from species accounts in [45]. In addition, a measure of relative brain mass, the encephalization quotient (EQ), was calculated for each species using a standard allometric equation of mammalian brain mass vs. body mass from [1]: EQ = brain mass/0.12 (body mass) 0.67. Absolute brain size, body size, maximum body size, and EQ were then log-transformed for statistical analysis (see below). Morphological data were deposited online in Supplemental Table S2.

We used the method of [25] to assess associations between $\omega$ and various phenotypic variables: absolute brain mass, absolute body mass, maximum body mass derived from [45], and EQ. For each of the 27 cetacean species that were scored for phenotypic data (see above), we calculated the average "root-to-tip" $\omega$ along branches extending from the last common ancestor of Cetacea to each extant cetacean species in our dataset. This approach has the advantage of producing a measure of selection that takes the entire evolutionary history of a lineage from a common ancestor into account and which is a property of the species tips in a way that is more comparable with extant phenotypes. This procedure also negates the issue of temporal effects on $\omega$ [25]. Root-to-tip $\omega$ values were estimated in PAML 4.0 [38] using a 2-rate branch model and the species tree of [42]. Phylogenetically controlled regression analyses of log-transformed root-to-tip $\omega$ versus each log-transformed morphological variable were performed using BayesTraits [46,47] and the time-calibrated tree of [42] to explicitly test for gene-phenotype associations. The significance of the regression analyses was determined using a one-tailed $t$-test in the positive direction, in order to test the hypothesis that there is a positive relationship between estimated selection pressure on MCPH1 ( $\omega)$ and the phenotypic variables as in [25]. Each regression was performed across all cetaceans and just within the Odontoceti.

In addition to the EQ, we also explored two other methods of assessing relative brain size. In the first method, we calculated residuals from a regression between brain and body mass and used these in a subsequent regression analysis with root-to-tip $\omega$. In the second method, we performed a multiple regression of brain and body mass with root-to-tip $\omega$. Both of these approaches produced similar results to the regression using the EQ, and are not shown here.

\section{Results}

\section{Characterization and Phylogenetic Analysis of $\mathrm{MCPH} 1$}

The alignment of the entire protein-coding region of MCPH1 consisted of $2571 \mathrm{bp}$ with 31 indels that were in frame and 3-33 bp (multiples of three) in length. Of the complete sequences, protein translations ranged from 822 amino acids (aa) in length in Mus to 842 aa in Callithrix. Phylogenetic analyses were performed using the exon 8 data set. The topology of the ML tree and the Bayesian consensus trees were congruent (Figure 2; Additional File 1: Figure S1, Figure S2). Most higherlevel relationships among mammalian orders and suborders were consistent with those of large comprehensive data sets $[39,40]$. Many higher-level relationships within Cetacea supported by MCPH1 (Figure 2) were congruent with the supermatrix of [42]; however, some relationships differed, especially within Delphinidae. For a more detailed description of the alignment and phylogenetic results, see Additional File 1.

\section{Molecular Evolution of $M C P H 1$}

For the $M C P H 1$ whole gene data set, site models that incorporated positive selection (M2a and M8) were significantly better fits to the data $(\mathrm{p}<0.001)$ than corresponding nearly neutral models (M1a and M8a). Overall, model M2a assigned $3.43 \%$ of codons to the class of positively selected sites $(\omega=2.334)$, and model M8 assigned $12.40 \%$ to the positive selection class $(\omega=$ 1.644). Both models M2a and M8 yielded three sites that had high probabilities ( $\geq 0.95$ in BEB analysis) of belonging to the class of sites with $\omega>1$ (308, 398, and 521), all of which were located in the IBS region between the BRCA1 C-terminal (BRCT) domains. None of the three positively selected sites showed evidence of convergence in amino acid sequence between primates and cetaceans. Analyses using branch models 


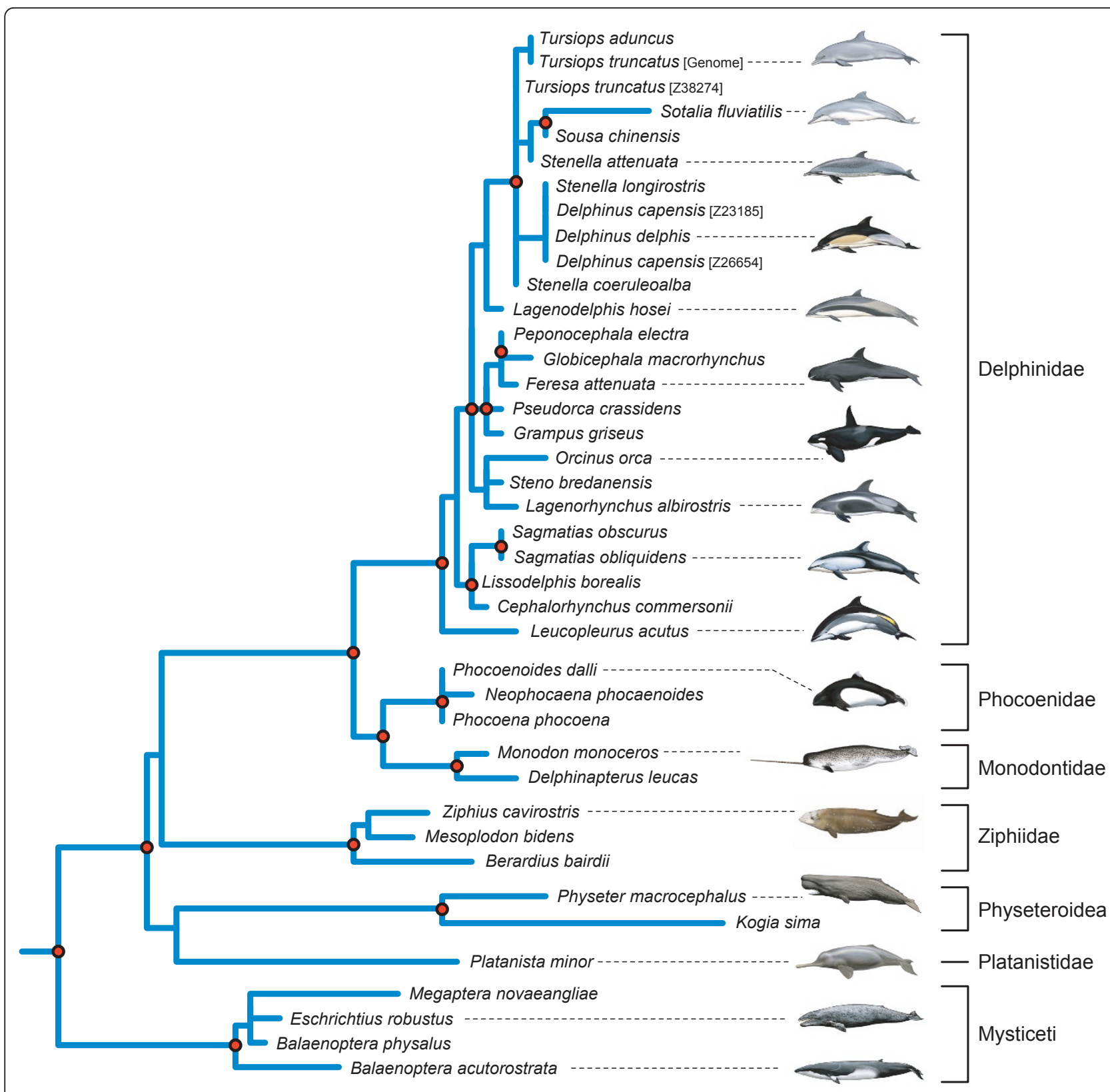

0.005 substitutions/site

Figure 2 Relationships among cetaceans in the maximum likelihood (ML) gene tree for MCPH1 based on the exon 8 data set. The phylogram of the whole tree that includes Primates and other terrestrial mammals is presented in Additional file 1, Figure S2. The cetacean part of the tree is illustrated here and shows well-supported nodes within Cetacea (red dots $=$ ML bootstrap $\geq 70 \%$ and Bayesian posterior probability (with and without indels) $\geq 0.95$ ). Higher level taxa are delimited by brackets to the right.

revealed that the free ratio model, in which each branch had a unique $\omega$, was significantly better $(p<$ $0.0001)$ than the model in which all branches were constrained to have the same $\omega$. The free-ratio model revealed two branches with $\omega>1$ : the branch leading to the LCA of Hominidae and the terminal branch leading to the bottlenose dolphin Tursiops (Figure 3).
The greatest $\omega$ was on the branch leading to the LCA of Hominidae $(\omega=3.022 ; 12.1$ nonsynonymous substitutions and 1.6 synonymous substitutions), and evidence of $\omega>1$ along this branch agrees with other studies $[16,19]$. The $\omega$ for the terminal branch leading to Tursiops $(1.271 ; 175.9$ nonsynonymous substitutions and 54.4 synonymous substitutions) is marginally 


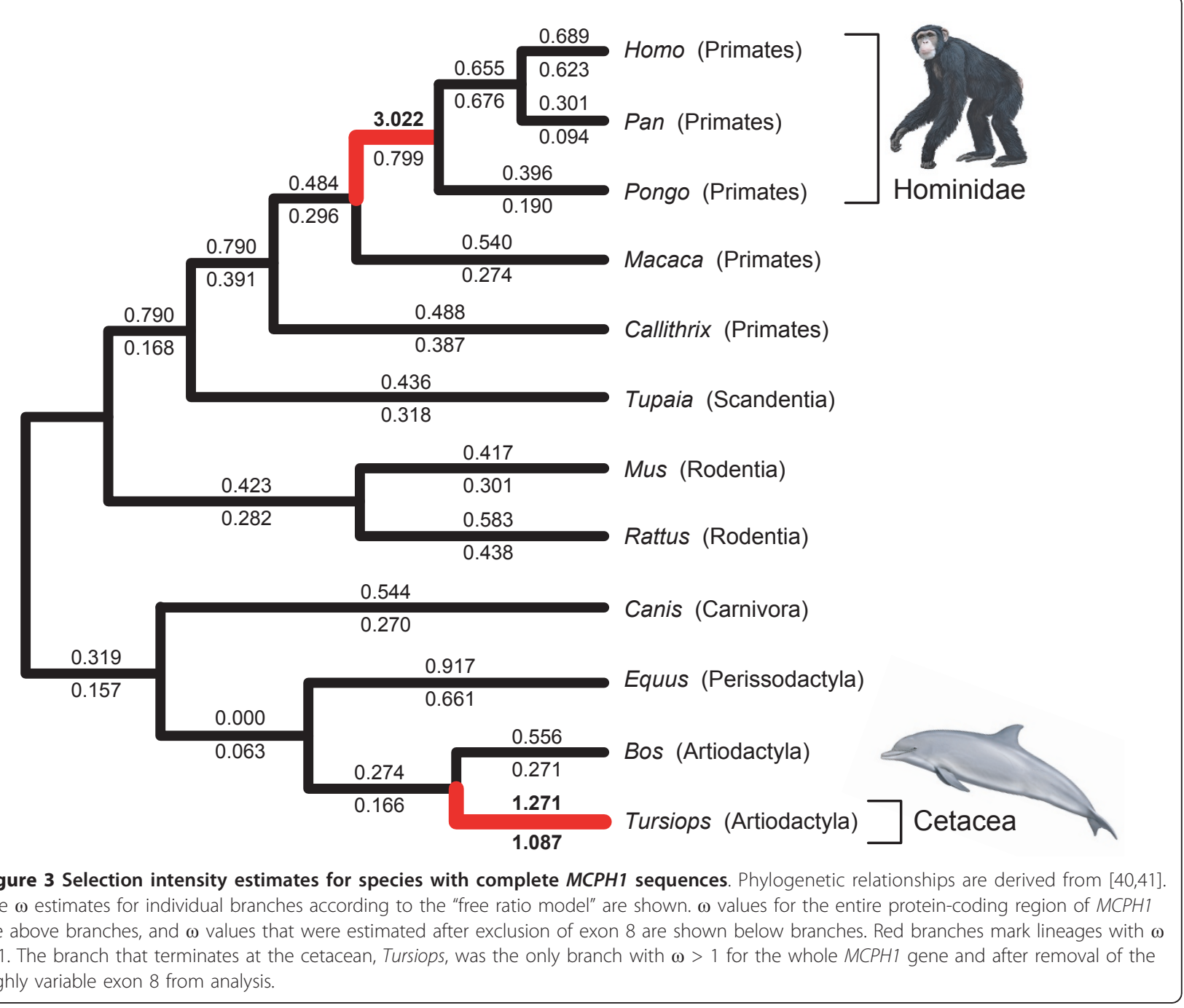

greater than the value expected for a complete absence of selective constraints $(\omega=1.000)$.

Using the coding sequences in the more taxon-rich exon 8 data set of all mammals, both site models incorporating positive selection (M2a and M8) again were significantly better (both $\mathrm{p}<0.001$ ) than corresponding models that only incorporate nearly neutral evolution (M1a and M8a; Table 1); This was true whether the species tree (Table 1) or the MCPH1 gene tree (Additional File 1: Table S3) was used (in the remainder of the paper, we refer primarily to results using the species tree; gene tree models were highly consistent with results using the species tree). The class of sites with $\omega$ $>1$ for the M8 model (14.0\% of sites) was characterized by an $\omega$ that was significantly greater than $1(\omega=1$. 721). M2a and M8 identified one and seven sites, respectively, with an $\omega>1$ using the BEB method (Table 1). Among sites identified as having an $\omega>1$, parallel changes in amino acid sequence were not shared between any cetacean and any primate. The mean $\omega$ for sites in Model M2a was $\omega=0.858$ for exon 8 . The reduced data set produced similar results (not shown) and identified the same sites under positive selection.

We performed further tests to determine whether evidence for positive selection was restricted to different subclades of our tree, or alternatively whether positive selection is a general feature that characterizes the evolution of $M C P H 1$ in the mammalian lineages sampled here. According to models M2a and M8, clades that showed strong evidence for positive selection acting at a subset of sites included Primates, Cetacea, Odontoceti (species tree only), Delphinidae, and all mammals excepting cetaceans and primates (M8 only) (Table 1). Both site models did not indicate positive selection within Mysticeti, but only four mysticete sequences were sampled here, and this is well below the recommended number for robust tests of positive selection using models M2a and M8 [38]. 
Table 1 Results for site model analyses using the species tree derived from multiple sources [39-42]

\begin{tabular}{cccccc}
\hline Model & -In $\mathbf{L}$ & Avg. & $\begin{array}{c}\text { Prop. sites } \\
\mathbf{\omega}>\mathbf{1}\end{array}$ & $\begin{array}{c}\boldsymbol{\omega} \text { sites with } \\
\mathbf{\omega}>\mathbf{1}\end{array}$ & $\begin{array}{c}\text { Sites under positive selection } \\
(\mathbf{p}>\mathbf{0 . 9 5}, \mathbf{B E B})\end{array}$ \\
\hline All mammals: & & & & \\
M1a & 15880.84 & 0.757 & & 2.063 & 362 \\
M2a & 15869.046 & 0.858 & 0.07 & 1.721 & $112,132,172,205,209,247,362$ \\
M8 & 15863.642 & 0.829 & 0.14 & &
\end{tabular}

M1a v. M2a: $d f=2,-2 \Delta \ln L=23.587, \mathbf{p}<0.001$

M8 v. M8a: $d f=1,-2 \Delta \ln L=26.804, \mathbf{p}<\mathbf{0 . 0 0 1}$

$\begin{array}{cc}\text { All cetaceans: } & \\ \text { M1a } & 3913.4037 \\ \text { M2a } & 3903.628 \\ \text { M8 } & 3903.6499 \\ \text { M8a } & 3913.4114\end{array}$

M1a v. M2a: $d f=2,-2 \Delta \ln L=19.551, \mathbf{p}<0.001$ M8 v. M8a: $d f=1,-2 \Delta \ln L=19.523, \mathbf{p}<\mathbf{0 . 0 0 1}$

$\begin{array}{cr}\text { All odontocetes: } & \\ \text { M1a } & 3519.5486 \\ \text { M2a } & 3514.0255 \\ \text { M8 } & 3514.0265 \\ \text { M8a } & 3519.5486 \\ \text { v. M2a: } d f=2,-2 \Delta \ln L=11.046, \mathbf{p}<\mathbf{0 . 0 0}\end{array}$

M1a v. M2a: $\mathrm{df}=2,-2 \Delta \mathrm{lnL}=11.046, \mathbf{p}<\mathbf{0 . 0 0 1}$

M8 v. M8a: $d f=1,-2 \Delta \ln L=11.044, \mathbf{p}<0.001$

$\begin{array}{cr}\text { All delphinids: } & \\ \text { M1a } & 2251.6189 \\ \text { M2a } & 2236.8709 \\ \text { M7 } & 2251.7481 \\ \text { M8 } & 2236.871 \\ \text { M8a } & 2251.6189 \\ \text { M2a: } d f=2,-2 \Delta l n L=29.496, \mathbf{p}<0.00\end{array}$

M1a v. M2a: $d f=2,-2 \Delta \ln L=29.496, \mathbf{p}<0.001$

M8 v. M8a: $d f=1,-2 \Delta \ln L=29.496, \mathbf{p}<\mathbf{0 . 0 0 1}$

$\begin{array}{cc}\text { All mysticetes: } & \\ \text { M1a } & 1690.448 \\ \text { M2a } & 1688.2783 \\ \text { M8 } & 1690.448 \\ \text { M8a } & 1690.448\end{array}$

M1a v. M2a: $d f=2,-2 \Delta \mathrm{ln} L=4.339, p=0.114$

M8 v. M8a: $d f=1,-2 \Delta \ln L=0, p=1.0$

\section{All primates:}

$\begin{array}{ll}\text { M1a } & 5103.5715 \\ \text { M2a } & 5098.7948 \\ \text { M8 } & 5098.9315 \\ \text { M8a } & 5103.5982\end{array}$

M1a v. M2a: $\mathrm{df}=2,-2 \Delta \ln L=9.553, \mathbf{p}=\mathbf{0 . 0 0 8}$

M8 v. M8a: $d f=1,-2 \Delta \ln L=9.333, \mathbf{p}=\mathbf{0 . 0 0 5}$
0.7975

0.9548

0.9458

0.8017

0.021

6.767

89,362

0.072

6.564

89,362

0.7351

0.9211

0.046

4.583

89

0.923

0.045

4.635

89,195

0.7351
0.548

0.9868

0.5

0.9887

0.5479
0.028

0.045

0.014

0.014
1.5764

1
41.761

41.757

None

$\begin{array}{ll}14.257 & 20,89,195 \\ 14.308 & 20,89,195\end{array}$

None
0.6245

0.728

0.7297

0.6244

\subsection{5}

0.132
2.608

2.13
None

209, 309 


\begin{tabular}{|c|c|c|c|c|c|}
\hline \multicolumn{6}{|c|}{ No cetaceans/primates: } \\
\hline M1a & 8990.4108 & 0.7309 & & & \\
\hline $\mathrm{M} 2 \mathrm{a}$ & 8987.9053 & 0.8132 & 0.059 & 2.17 & None \\
\hline M8a & 8986.2851 & 0.7017 & & & \\
\hline \multicolumn{6}{|c|}{ M1a v. M2a: $d f=2,-2 \Delta \ln L=5.011, p=0.082$} \\
\hline M8 v. M8a: $d f=$ & $p=0.002$ & & & & \\
\hline
\end{tabular}

Each data set is listed separately with models, likelihood score $(-\ln L)$, average $\omega$, the proportion of sites in the site class with $\omega>1$, the $\omega$ estimate for the site class with $\omega>1$, and the specific sites with $\omega>1$ using the Bayes empirical Bayes (BEB) procedure. Likelihood ratio tests for site models are also shown below for each data set with degrees of freedom $(\mathrm{df})$, likelihood ratio $(-2 \Delta \ln L)$, and $p$-value. Statistically significant $p$-values are shown in bold.

For the exon 8 data set, several branch model comparisons were executed (Table 2). We compared model M0 (one $\omega$ across the whole tree) to the free-ratio model in which all branches had separate $\omega$ values; the parameter rich free-ratio model was not a significantly better fit than M0 ( $p=0.464$; Table 2). Of the two-branch models explored here (see Methods), three "foreground" branches or sets of foreground branches showed evidence of $\omega$ greater than 1: the branch leading to the LCA of Mysticeti $(\omega=1.340 ; \omega=3.115$ for the gene tree), the set of all mysticete branches $(\omega=1.838)$, and the terminal branch that connects to Physeter $(\omega=$ 1.447). Although $\omega$ was high for foreground branches in these various two branch models, fit was not significantly better than model M0 (one $\omega$ ) following Bonferroni corrections. In mysticetes, $\omega$ was generally high but not significantly greater than 1 according to the LRT (Table 2).

\section{MCPH1 Polymorphism in Cetacea}

A total of 38 sites in exon 8 were heterozygous in at least one of the cetacean species sampled here; 14

Table 2 Branch models

\begin{tabular}{lcccccc}
\hline Model & $-\ln \boldsymbol{L}$ & $\boldsymbol{p}$ (GT) & $\boldsymbol{\omega}(\mathrm{GT})$ & $-\ln \boldsymbol{L}$ & $\boldsymbol{p}(\mathrm{ST})$ & $\begin{array}{c}\boldsymbol{\omega} \\
(\mathrm{ST})\end{array}$ \\
\hline Cetacea & 15867.095 & 0.534 & 0.963 & 15996.857 & 0.545 & 0.962 \\
Mysticeti & 15865.373 & 0.050 & 3.115 & 15996.264 & 0.213 & 1.340 \\
Odontoceti & 15867.250 & 0.781 & 0.639 & 15997.024 & 0.858 & 0.767 \\
Physeter & 15867.035 & 0.477 & 1.328 & 15996.698 & 0.408 & 1.447 \\
Delphinoidea & 15867.288 & 0.994 & 0.768 & 15997.023 & 0.854 & 0.785 \\
Delphinidae & 15867.268 & 0.840 & 0.730 & 15996.491 & 0.295 & 0.440 \\
Orcinus & 15866.947 & 0.409 & 0.428 & 15996.963 & 0.695 & 0.613 \\
All cetaceans & 15865.290 & 0.046 & 0.940 & 15995.134 & 0.051 & 0.930 \\
All mysticetes & 15864.062 & 0.011 & 1.840 & 15993.772 & 0.011 & 1.838 \\
All odontocetes & 15866.791 & 0.319 & 0.861 & 15996.583 & 0.340 & 0.854 \\
All delphinoids & 15867.261 & 0.816 & 0.789 & 15997.020 & 0.840 & 0.759 \\
All delphinids & 15867.026 & 0.469 & 0.899 & 15996.952 & 0.675 & 0.829 \\
Free ratio & 15797.540 & 0.310 & & 15929.589 & 0.464 & \\
\hline
\end{tabular}

These are listed with likelihood score $(-\ln L), p$-value of likelihood ratio test $v$. $\mathrm{MO}$, and $\omega$ for the foreground branches when applicable. Results are shown for analyses using the MCPH1 gene tree as well as the species tree. species showed allelic variation. Parsimony optimization of this variation onto the $M C P H 1$ gene tree (Figure 2) suggests that 22 nonsynonymous and 18 synonymous changes can account for the intraspecific variation (28 transitions and 12 transversions). None of the 40 point mutations included changes to a stop codon. Ten changes were at first codon positions, 10 at second positions, and 20 at third positions; some of the allelic differences were shared among closely related species in our sample (e.g., between Delphinus delphis and D. capensis).

A comparison between substitutions that characterize change among cetacean species and polymorphic mutations within cetacean species indicated that a greater proportion of substitutions among species were nonsynonymous than within species (326 nonsynonymous and 154 synonymous estimated using model M0 for the species tree of [42] versus 22 nonsynonymous and 18 synonymous changes within species). The larger proportion of nonsynonymous change across the cetacean phylogeny (68\%) relative to the proportion of nonsynonymous mutations within species $(55 \%)$ is consistent with positive selection [48], but much more extensive sampling of $M C P H 1$ variation within species is necessary to rigorously test this hypothesis in the future.

\section{Association of $\omega$ with Phenotype}

We explicitly tested the hypothesis that selection intensity on $M C P H 1$, as represented by root-to-tip $\omega$ (Additional File 1: Table S2), was positively correlated with different measures of brain and body size in cetaceans. Based on phylogenetically controlled regression analyses, we found no significant association between $\omega$ and absolute brain mass or EQ (Table 3). There is a significant association with absolute body mass, $(\mathrm{p}=0.024$; Table 3$)$ and a non-significant trend with brain mass across all cetaceans, which suggests a closer relationship between $\omega$ and overall body size. However both regression coefficients are low, and when mysticetes are excluded the associations become weaker, implying that these largebodied species have a major effect on results (Table 3). To further explore the relationship between selection on 


\begin{tabular}{|c|c|c|c|c|c|c|}
\hline \multicolumn{7}{|l|}{ A) } \\
\hline Analysis & $\mathbf{n}$ & $\mathrm{R}^{2}$ & t-statistic & & $\begin{array}{c}\text { p-value } \\
\text { (1 tailed positive) }\end{array}$ & \\
\hline \multicolumn{7}{|l|}{ All Cetaceans } \\
\hline$\omega$ vs. brain mass & 27 & 0.100 & 1.663 & & 0.054 & \\
\hline$\omega$ vs. EQ & 27 & 0.105 & -1.710 & & 1.000 & \\
\hline$\omega$ vs. body mass & 27 & 0.148 & 2.087 & & 0.024 & \\
\hline$\omega$ vs. max body mass & 27 & 0.112 & 1.776 & & 0.044 & \\
\hline \multicolumn{7}{|l|}{ Toothed Whales only } \\
\hline$\omega$ vs. brain mass & 25 & 0.064 & 1.249 & & 0.112 & \\
\hline$\omega$ vs. EQ & 25 & 0.031 & -0.860 & & 1.000 & \\
\hline$\omega$ vs. body mass & 25 & 0.079 & 1.408 & & 0.086 & \\
\hline$\omega$ vs. max body mass & 25 & 0.084 & 1.454 & & 0.079 & \\
\hline B) & & & \multicolumn{2}{|c|}{$\mathrm{dN}$} & \multicolumn{2}{|r|}{ dS } \\
\hline Analysis & $\mathrm{n}$ & $\mathrm{R}^{2}$ & t-statistic & $\begin{array}{c}\text { p-value } \\
\text { (1 tailed positive) }\end{array}$ & t-statistic & $\begin{array}{c}p \text {-value } \\
\text { (1 tailed negative) }\end{array}$ \\
\hline \multicolumn{7}{|l|}{ All Cetaceans } \\
\hline Body mass vs. $d N \& d S$ & 27 & 0.231 & 0.803 & 0.215 & -2.498 & 0.010 \\
\hline Brain mass vs. $d N \& d S$ & 27 & 0.173 & 0.521 & 0.304 & -2.022 & 0.027 \\
\hline
\end{tabular}

A) Regression analyses using BayesTraits of root-to-tip $\omega(d N / d S)$ versus absolute brain mass, relative brain mass (encephalization quotient $=$ EQ), absolute body mass, and maximum body mass derived from [45]. Results are shown for all cetaceans and toothed whales only. B) Multiple regression analyses of absolute brain mass and absolute body mass versus $d N$ (rate of nonsynonymous substitution) and $d S$ (rate of synonymous substitution).

$M C P H 1$ and phenotypic evolution, we performed a multiple regression with root-to-tip $d N$ and root-to-tip $d S$ (log-transformed) for both absolute body and brain mass across cetaceans, in order to partition out the effects of $d N$ and $d S . d N$ was not significantly positively associated with either phenotype (body mass: $t_{23}=0.803, p=0.215$; brain mass $\left.\mathrm{t}_{23}=0.521, \mathrm{p}=0.304\right)$, whereas $d S$ was significantly negatively associated with both (body mass: $\mathrm{t}_{23}=$ 2.498, $\mathrm{p}=0.010$; brain mass $\mathrm{t}_{23}=2.022, \mathrm{p}=0.027$ ). The negative relationship between $d S$ and body mass is consistent with the conclusions of previous studies which show that the neutral rate of molecular evolution is associated with body mass and life history traits such as generation time and life span $[49,50]$. Hence, we cannot rule out the possibility that the weak associations observed reflect variation in the neutral substitution rate, which is associated with body size, rather than adaptive evolution.

\section{Discussion}

\section{MCPH1 as a Phylogenetic Marker in Mammals}

Despite, or because of its molecular evolutionary dynamics, exon 8 of $M C P H 1$ performed well as a phylogenetic marker at both deep and shallow nodes (Figure 2; Additional File 1: Figure S1, Figure S2). Most relationships among mammalian orders and supraordinal clades in the $M C P H 1$ tree are congruent with analyses supported by much larger data sets $[39,40]$, and 15 clades within Primates are characterized by high support scores and congruence with comprehensive phylogenetic hypotheses for this group [41,51] (Additional File 1: Figure S2). We also obtained good resolution within Cetacea (Figure 2), as compared to a recent supermatrix analysis of data from over 50 genes [42]. Overall, the congruence of our MCPH1 topology (Figure 2; Additional File 1: Figure S1, Figure S2) with published results is impressive, and is consistent with some previous studies which showed that highly variable nuclear genes with extensive amino acid replacements and evidence of positive selection can be efficient phylogenetic markers $[52,53]$.

\section{The Evolution of $M C P H 1$ across Mammals}

Cetaceans, especially odontocetes, display multiple neuroanatomical and behavioral similarities with primates. Representatives of both groups have evolved large brains relative to their body sizes as well as highly complex cognitive abilities $[9,10]$, although some researchers dispute this latter point [13]. In addition, cetaceans show similarities with great apes in brain histology, neural connectivity, and enlargement of specific parts of the brain associated with cognition and social awareness [54], as well as extensive gyrification, or folding of the cerebral cortex $[10,54]$. Due to their convergence in multiple neurological and behavioral features, cetaceans present an obvious test of the hypothesis that $\mathrm{MCPH} 1$ is related to the evolution of large brain size in mammals.

Analysis of the full coding sequence of MCPH1 (Figure 3) revealed high $\omega$ s on the stem branch to 
hominid primates and also on the terminal branch leading to the bottlenose dolphin Tursiops. Very high average $\omega$ over the entire protein-coding sequence of a gene, especially one this long ( $2500 \mathrm{bp})$, is rare, as indicated by comparisons across complete genome sequences of different species (e.g., [55]). The high $\omega$ on the cetacean branch, in combination with the superior fit of positive selection models to the $M C P H 1$ data relative to nearly neutral models, suggests a persistent signal of positive selection since the bottlenose dolphin lineage split from Bos taurus (Ruminantia). Comparative analyses of $M C P H 1$ sequences did not reveal evidence for precise site-specific convergent molecular evolution of $M C P H 1$ between cetaceans and primates at the amino acid level in either the whole gene or exon 8 data sets. However for exon 8 , seven sites were identified as evolving under positive selection in Boreoeutheria (Table 1). Changes at positively selected sites, inferred using the site models, were not restricted to either Primates or Cetacea suggesting that the gene may have an important evolutionary role across mammals (Table 1). Further detailed analysis of other mammalian clades will be needed to determine how extensive positive selection has been on MCPH1.

\section{The Evolution of $M C P H 1$ within Cetacea and its Relation to Brain Size}

With the inclusion of more species in the exon 8 data set, estimated $\omega$ ratios revealed a more complex pattern of evolution in cetaceans. In general, exon 8 had a relatively high mean $\omega$ across mammals (Table 1 ) and $\omega$ within Cetacea was elevated relative to the remaining branches in the tree (Table 2). Both Odontoceti and Delphinoidea represent clades in which shifts to larger relative brain sizes have been proposed, and the highest EQs are restricted to members of Delphinidae [10]. However, our comparative analyses of $M C P H 1$ did not reveal especially high $\omega$ scores on the branches leading to the LCAs of Cetacea, Odontoceti, Delphinoidea, or Delphinidae (Table 2). Absence of strong evidence for positive selection in these lineages does not match the evolutionary change of the EQ in which there is a large increase in relative brain size along the lineage leading from the LCA of Cetacea to the LCA of Delphinidae [10]. Regression analyses agreed with these results, finding no robust associations between $\omega$ and absolute or relative brain size (Table 3 ).

Some of the largest $\omega$ scores, according to the branch models, were recorded along the branch leading to the LCA of mysticetes and across all mysticete branches (Table 2). This is contrary to the hypothesis that selection in $M C P H 1$ is related to an increase in relative brain size $[16,19]$, as mysticetes have smaller brains than expected given their body size compared to the mammalian average [2]; however, it does not rule out that $\mathrm{MCPH1}$ may be linked to overall change in EQ within cetaceans. Although mysticetes have low EQs, the brains of these cetaceans have the largest absolute mass in the animal kingdom, aside from elephants and the odontocete Physeter macrocephalus (giant sperm whale; Figure 1) [10]. It has been argued that the molecular evolution of genes involved in the proliferation of neural progenitor cells should bear a closer relation to absolute brain size than relative brain size [25]; if $M C P H 1$ has had such an evolutionary role we may then expect it to have high rates of evolution during the evolution of the largest cetaceans (Figure 1). Both the ancestral mysticete branch and the Physeter terminal branch have high $\omega$ (Table 2), but the branch leading to the very large delphinid, Orcinus orca which dwarfs most extant and extinct oceanic dolphins (Figure 1), did not show an extreme $\omega$ for $M C P H 1$ in comparison to dolphins with much smaller brain and body sizes (Table 2; Additional File 1: Table S2). Our regression analyses do not support a robust association of $\omega$ with absolute brain mass, so we cannot confidently say that the high $\omega$ in mysticetes is causally related to selection for large brain size. Indeed, a stronger association was found between root-to-tip $\omega$ and body mass and therefore we cannot exclude body mass, or other correlated traits, from being the relevant phenotype. Our multiple regressions with $d N$ and $d S$ furthermore suggest that these trends may be driven largely by variation in $d S$, instead of $d N$, perhaps related to variation in life history traits which affect the rate at which neutral variants are fixed $[49,50]$.

The lack of association between brain size and the evolution of MCPH1 found in cetaceans agrees with the conclusions drawn from a recent study of anthropoid primates which showed that $M C P H 1$ is not associated with the evolution of either absolute or relative brain size [25], despite the clear critical importance of MCPH1 in brain development. Together these studies question the commonly held assumption that this locus has a direct role in the evolution of brain size as a gross measure $[16,19,56]$. While we cannot rule out more nuanced roles in brain evolution, the phenotypic relevance of positive selection on this locus is currently an outstanding issue.

MCPH1 is expressed in many other tissues [32], and mutations in $M C P H 1$ have been discovered in cancerous tumors [27]. Some mysticetes have very long lifespans [57], are the largest animals that have yet evolved, and are characterized by a very high fetal growth rate [58]. Given that $M C P H 1$ functions in cell cycle regulation and DNA damage repair [24], it is plausible that positive selection in mysticetes and other mammals is related to the evolution of other phenotypes such as the rate of 
growth, absolute number of cells, or tumor suppression in very large organisms with long lifespans, although these hypotheses are currently speculative. Some evidence, presented here, suggests that positive selection has acted on this locus in non-primate, non-cetacean mammals (Table 1 - model M8). It may therefore be possible to study the evolutionary function of MCPH1, and to test these hypotheses, in more experimentally tractable organisms such as rodents.

\section{Conclusions}

Extant cetacean species are characterized by a very broad range of body sizes and brain sizes, among the greatest variation seen in any mammalian order (Figure 1). Although this study did not find evidence for a statistically significant association between selection intensity $(\omega)$ in $M C P H 1$, a gene associated with microcephaly, and absolute or relative brain size in cetaceans (Table 3), some intriguing patterns emerged from detailed phylogenetic and molecular analyses of $M C P H 1$. Evolutionary models that included parameters for positive selection consistently fit the $\mathrm{MCPH} 1$ sequence data significantly better than alternative models of negative selection and neutral change (Table 1). Using complete sequences of $M C P H 1$, we identified $\omega>1$ in only two cases, along the lineage leading to Tursiops (bottlenose dolphin) and also on the branch leading to Hominidae (human, gorilla, chimp, and orang). Furthermore, analysis of a larger data set of cetacean sequences derived from the hypervariable exon 8 again indicated a very high average $\omega$ within Cetacea, with the largest $\omega$ values in Mysticeti, a group characterized by low EQ but large absolute brain and body weights. Despite finding strong evidence for positive selection having acted on a gene which has a key role in brain development, we found no compelling evidence to support the hypothesis that there is an association between the evolution of this locus and the evolution of brain size in cetaceans. This is in agreement with results from anthropoid primates, and suggests that positive selection on $M C P H 1$ may be related to change in phenotypes other than gross brain size.

\section{Additional material}

Additional file 1: Supplemental Information. This includes

Supplemental Methods, Supplemental Results, Supplemental Appendix I, Supplemental Tables S1, S2, S3 and Supplemental Figures S1, S2.

\section{Acknowledgements}

Funding was provided by NSF grants to JG (DEB-0743724, DEB-0640313) and a BBSRC student fellowship to SHM. We thank P. Morin, K. Robertson, S. Chivers, A. Dizon, R. LeDuc, T. Jefferson, G. Braulik, H. Rosenbaum, P. Palsbøll, M. Stanhope, M. Springer, M. Milinkovitch, Southwest Fisheries Science Center, The Marine Mammal Center, Mote Marine Laboratory, Wildlife
Conservation Society, World Wildlife Fund, and Smithsonian Institution Division of Mammals for providing DNA samples. R. Meredith, J. Starrett, M. Springer, and C. Hayashi provided helpful comments on previous drafts of this manuscript. C. Buell executed paintings of cetaceans.

\section{Author details}

'Department of Biology, University of California, Riverside, Riverside, CA 92521 USA. ${ }^{2}$ Center for Molecular Medicine and Genetics, Wayne State University School of Medicine, 540 E. Canfield Ave., Detroit, Ml 48201 USA. ${ }^{3}$ Department of Zoology, University of Cambridge, Downing Street, Cambridge, CB2 3EJ, UK.

\section{Authors' contributions}

MM, CC, and JG gathered MCPH1 sequence. MM aligned sequences and conducted phylogenetic analyses. MM and SM executed PAML analyses. SM conducted regression analyses. MM wrote the paper, and all authors read, edited, and approved the final manuscript.

Received: 27 October 2010 Accepted: 14 April 2011

Published: 14 April 2011

\section{References}

1. Jerison $\mathrm{JH}$ : Evolution of the Brain and Intelligence. New York: Academic Press; 1973.

2. Worthy GA, Hickie JP: Relative brain size in marine mammals. Am Nat 1986, 128:445-459.

3. Marino L: A comparison of encephalization between odontocete cetaceans and anthropoid primates. Brain Behav Evol 1998, 51:230-238.

4. Oelschläger HHA: The dolphin brain-a challenge for synthetic neurobiology. Brain Res Bull 2008, 75:450-459.

5. Lefebvre L, Reader SM, Sol D: Brains, innovations and evolution in birds and primates. Brain Behav Evol 2004, 63:233-246

6. Healy SD, Rowe $C$ : A critique of comparative studies of brain size. Proc $R$ Soc B 2007, 274:453-464

7. Deaner RO, Isler K, Burkhart J, van Schaik C: Overall brain size, and not encephalization quotient, best predicts cognitive ability across nonhuman primates. Brain Behav Evol 2007, 70:115-124.

8. Marino L, McShea D, Uhen MD: The origin and evolution of large brains in toothed whales. Anat Rec 2004, 281A:1247-1255.

9. Marino L: Brain size evolution. In Encylcopedia of Marine Mammals. Edited by: Perrin WF, Wursig B, Thewissen JGM. San Diego: Academic Press; 2002:158-162.

10. Marino L, Connor RC, Fordyce RE, Herman LM, Hof PR, Lefebvre L, Lusseau D, McCowan B, Nimchinsky EA, Pack AA, Rendell L, Reidenberg JS, Reiss D, Uhen MD, Van der Gucht E, Whitehead H: Cetaceans have complex brains for complex cognition. PLOS Biol 2007, 5:e139.

11. Jerison $\mathrm{JH}$ : Brain and intelligence in whales. In Whales and Whaling. Volume 2. Edited by: Frost S. Canberra: C. J. Thomson, Australian Commonwealth Government Printer; 1978:159-197.

12. Connor RC, Mann J, Tyack PL, Whitehead H: Social evolution in toothed whales. Trends Ecol Evol 1998, 13:228-232.

13. Manger PR: An examination of cetacean brain structure with a novel hypothesis correlating thermogenesis to the evolution of a big brain. Biol Rev 2006, 81:293-338.

14. Zhang J: Evolution of the human ASPM gene, a major determinant of brain size. Genetics 2003, 165:2063-2070.

15. Dorus S, Vallender EJ, Evans PD, Anderson JR, Gilbert SL, Mahowald M Wyckoff GJ, Malcom CM, Lahn BT: Accelerated evolution of nervous system genes in the origin of Homo sapiens. Cell 2004, 119:1027-1040.

16. Evans PD, Anderson JR, Vallender EJ, Choi SS, Lahn BT: Reconstructing the evolutionary history of microcephalin, a gene controlling human brain size. Hum Mol Genet 2004, 13:1139-1145.

17. Evans PD, Anderson JR, Vallender EJ, Gilbert SL, Malcom CM, Dorus S, Lahn BT: Adaptive evolution of ASPM, a major determinant of cerebral cortical size in humans. Hum Mol Genet 2004, 13:489-494.

18. Kouprina N, Pavlicek A, Mochida GH, Solomon G, Gersch W, Yoon YH, Collura R, Ruvolo M, Barrett JC, Woods CG, Walsh CA, Jurka J, Larlonov : Accelerated evolution of the ASPM gene controlling brain size begins prior to human brain expansion. PLOS Biol 2004, 2:653-663.

19. Wang Y, Su B: Molecular evolution of microcephalin, a gene determining human brain size. Hum Mol Genet 2004, 13:1131-1137. 
20. Evans PD, Vallender EJ, Lahn BT: Molecular evolution of the brain size regulator genes CDK5RAP2 and CENPJ. Gene 2006, 375:75-79.

21. Kumar A, Girimaji SC, Duvvari MR, Blanton SH: Mutations in STIL, encoding a pericentriolar and centrosomal protein cause primary microcephaly. Am J Hum Genet 2009, 84:286-290.

22. Yu TW, Mochida GH, Tischfield DJ, Sgaier SK, Flores-Sarnat L, Sergi CM, Topçu M, McDonald MT, Barry BJ, Felie JM, Sunu C, Dobyns WB, Folkerth RD, Barkovich JA, Walsh CA: Mutations in WDR62, encoding a centrosome-associated protein, cause microcephaly with simplified gyri and abnormal cortical architecture. Nat Genet 2010, 42:1015-1020.

23. Vallender EJ, Mekel-Bobrov N, Lahn BT: Genetic basis of human brain evolution. Trends Neurosci 2008, 31:637-644.

24. Thornton GK, Woods CG: Primary microcephaly: do all roads lead to Rome? Trends Genet 2009, 25:501-510.

25. Montgomery SH, Capellini I, Venditti C, Barton RA, Mundy NI: Adaptive evolution of four microcephaly genes and the evolution of brain size in anthropoid primates. Mol Biol Evol 2011, 28:625-638.

26. Lin SY, Rai R, Li K, Xu ZX, Elledge SJ: BRIT/MCPH1 is a DNA damage responsive protein that regulates the Brca1-Chk1 pathway, implicating checkpoint dysfunction in microcephaly. Proc Nat Acad Sci USA 2005, 102:15105-15109.

27. Rai R, Dal H, Multani AS, Li K, Chin K, Gray J, Lahad JP, Liang J, Mills GB, Meric-Bernstam F, Lin SY: BRIT1 regulates early DNA damage response, chromosomal integrity, and cancer. Cancer Cell 2006, 10:145-157.

28. Wood JL, Liang Y, Li K, Chen J: Microcephalin/MCPH1 associates with the Condensin II complex to function in homologous recombination repair. J Biol Chem 2008, 283:29586-29592.

29. Peng G, Yim EK, Dai H, Jackson AP, van der Burgt I, Pan MR, Hu R, Li K, Lin SY: BRIT1/MCPH1 links chromatin remodeling to DNA damage response. Nat Cell Biol 2009, 11:865-872.

30. Wu X, Mondal G, Wang X, Wu J, Yang L, Pankratz VS, Rowley M, Couch FJ: Microcephalin regulates BRCA2 and Rad51-associated DNA doublestrand break repair. Cancer Res 2009, 69:5531-5536.

31. Liang Y, Gao H, Lin SY, Peng G, Huang X, Zhang P, Goss JA, Brunicardi FC, Multani AS, Chang S, Li K: BRIT1/MCPH1 is essential for mitotic and meiotic recombination DNA repair and maintaining genomic stability in mice. PLoS Genet 2010, 6:e1000826.

32. Jackson AP, Eastwood H, Bell SM, Adu J, Toomes C, Carr IM, Roberts E, Hampshire DJ, Crow YJ, Mighell AJ, Karbani G, Jafri H, Rashid Y, Mueller RF, Markham AF, Woods CG: Identification of microcephalin, a protein implicated in determining the size of the human brain. Am J Hum Genet 2002, 71:136-142.

33. Woods CG, Bond J, Enard W: Autosomal recessive primary microcephaly (MCPH): a review of clinical, molecular, and evolutionary findings. Am J Hum Genet 2005, 76:717-728.

34. Cox J, Jackson AP, Bond J, Woods CG: What primary microcephaly can tell us about brain growth. Trends Mol Med 2006, 12:358-366.

35. O'Driscoll M, Jeggo P: The role of double-strand break repair-insights from human genetics. Nat Rev Genet 2006, 7:45-54.

36. Goodman M, Sterner KN, Islam M, Uddin M, Sherwood CC, Hof PR, Hou ZC, Lipovich L, Jia H, Grossman LI, Wildman DE: Phylogenomic analyses reveal convergent patterns of adaptive evolution in elephant and human ancestries. Proc Nat Acad Sci USA 2009, 106:20824-20829.

37. Thompson JD, Higgins DG, Gibson TJ: CLUSTAL W: improving the sensitivity of progressive multiple sequence alignment through sequence weighting, position-specific gap penalties and weight matrix choice. Nucleic Acids Res 1994, 22:4673-4680.

38. Yang Z: PAML 4: a program package for phylogenetic analysis by maximum likelihood. Mol Biol Evol 2007, 24:1586-1591.

39. Murphy WJ, Eizirik E, Johnson WE, Zhang YP, Ryder OA, O'Brien SJ: Molecular phylogenetics and the origins of placental mammals. Nature 2001, 409:614-618.

40. Springer MS, Burk-Herrick A, Meredith R, Eizirik E, Teeling E, O'Brien SJ, Murphy WJ: The adequacy of morphology for reconstructing the early history of placental mammals. Syst Biol 2007, 56:673-684.

41. Fabre PH, Rodrigues A, Douzery EJP: Patterns of macroevolution among Primates inferred from a supermatrix of mitochondrial and nuclear DNA. Mol Phylogenet Evol 2009, 53:808-825.

42. McGowen MR, Spaulding M, Gatesy J: Divergence date estimation and a comprehensive molecular tree of extant cetaceans. Mol Phylogenet Evol 2009, 53:891-906.
43. Schwerdtfeger WK, Oelschläger HA, Stephan H: Quantitative neuroanatomy of the brain of the La Plata dolphin, Pontoporia blainvillei. Anat Embryol 1984, 170:11-19.

44. Tarpley RJ, Ridgway SH: Corpus callosum size in delphinid cetaceans. Brain Behav Evol 1994, 44:156-165.

45. Jefferson TA, Webber MA, Pitman RL: Marine Mammals of the World: A Comprehensive Guide to their Identification. London: Academic Press: 2008.

46. Pagel M: Inferring the historical patterns of biological evolution. Nature 1999, 401:877-884.

47. Pagel M, Meade A, Barker D: Bayesian estimation of ancestral character states on phylogenies. Syst Biol 2004, 53:673-684.

48. McDonald JH, Kreitman M: Adaptive protein evolution at the Adh locus in Drosophila. Nature 1991, 351:652-654.

49. Nikolaev SI, Montoya-Burgos Jl, Popadin K, Parand L, Margulies EH, National Institutes of Health Intramural Sequencing Center Comparative Sequencing Program, Antonarakis SE: Life-history traits drive the evolutionary rates of mammalian coding and noncoding genomic elements. Proc Nat Acad Sci USA 2007, 104:20443-20448.

50. Lartillot N, Poujol R: A phylogenetic model for investigating correlated evolution of substitution rates and continuous phenotypic characters. Mol Biol Evol 2011, 28:729-744.

51. Chatterjee HJ, Ho SYW, Barnes I, Groves C: Estimating the phylogeny and divergence times of primates using the supermatrix approach. BMC Evol Biol 2009, 9:259.

52. Jansa SA, Forsman JF, Voss RS: Different patterns of selection on the nuclear genes IRBP and DMP-1 affect the efficiency but not the outcome of phylogeny estimation for didelphid marsupials. Mol Phylogenet Evol 2006, 38:363-380.

53. Gatesy J, Swanson W: Adaptive evolution and phylogenetic utility of $A C R$ (acrosin), a rapidly evolving mammalian fertilization gene. J Mammal 2007, 88:32-42.

54. Butti C, Sherwood CC, Hakeem AY, Allman JM, Hof PR: : Total number and volume of Von Economo neurons in the cerebral cortex of cetaceans. J Comp Neur 2009, 515:243-259.

55. Gibbs RA, Rate Genome Sequencing Project Consortium, et al: Genome sequence of the brown Norway rat yields insights into mammalian evolution. Nature 2004, 428:493-521.

56. Gilbert SL, Dobyns WB, Lahn BT: Genetic links between brain development and brain evolution. Nat Rev Genet 2005, 6:581-590.

57. George JC, Bada J, Zeh J, Scott L, Brown SE, O'Hara T, Suydam R: Age and growth estimates of bowhead whales (Balaena mysticetus) via aspartic acid racemization. Can J Zool 1999, 77:571-580

58. Frazer JFD, Huggett ASG: Specific foetal growth rates of cetaceans. J Zool Lond 1973, 169:111-126.

doi:10.1186/1471-2148-11-98

Cite this article as: McGowen et al:: Phylogeny and adaptive evolution of the brain-development gene microcephalin (MCPH1) in cetaceans. BMC Evolutionary Biology 2011 11:98.

\section{Submit your next manuscript to BioMed Central and take full advantage of:}

- Convenient online submission

- Thorough peer review

- No space constraints or color figure charges

- Immediate publication on acceptance

- Inclusion in PubMed, CAS, Scopus and Google Scholar

- Research which is freely available for redistribution 